\title{
Case Report on Forgotten Vaginal Pessary Leading to Incarceration
}

\author{
Dr. Shradha ${ }^{1}$, Dr (Prof.) P. B. Sahay ${ }^{2}$ \\ ${ }^{1} \mathrm{MD}$, Department of Obstetrics and gynecology, Rajendra Institute of Medical Sciences, Ranchi, \\ ${ }^{2}$ Professor and Head of department, MS, FICOG, Department of Obstetrics and gynecology, Rajendra Institute of Medical Sciences, Ranchi, \\ Jharkhand, India
}

\begin{abstract}
A ring vaginal pessary are most commonly used for conservative management of uterine fibroid in frail elderly and patient unfit for surgery. These are safe, easy to apply but needs regular and systematical follow up. Failing this there may be prolonged retention of pessary in vagina leading to wide spectrum of complications ranging from erosion to squamous cell carcinoma. Here we present a case of 65 years postmenopausal women presenting with foul smelling vaginal discharge and bleeding per vaginum raising to suspicion of genital tract malignancy. On examination, she was found to have incarcerated ring vaginal pessary which was removed under iv anaesthesia. Patient was asymptomatic thereafter. Areview of relevent literatures was undertaken and complicatins associated with vaginal pessaries are reviewed.
\end{abstract}

Keywords: vaginal pessary, pelvic organ prolapse, forgotten pessary.

\section{Introduction}

Pessaries i.e. a device inserted into vagina for correction of pelvic organ prolapse have been used since the times of Hippocrates (400 BC). He first used half a pomegranate soaked in wine into vagina. Since then, the journey of evolution of pessary underwent wide modifications. Modern pessaries are widely made up of silicone, latex rubber or acrylic. Amongst these, silicone has added advantage of being inert, flexible yet sturdy, non absorbent and autoclavable. Pessaries though evolved for nonsurgical management of POP, its used has been widened to conditions like urinary incompetence, uterine retroversion, cervical incompetence and more recently for local administration of hormones.

Pessary application is indeed cheap and effective measure for management of POP in elderly and frail who are unfit for surgery, but proper follow up is needed. Forgotten pessaries may lead to complications like persistent foul smelling vaginal discharge, vaginal erosions, pessary incarceration, fistula, herniation, uraemia and even squamous cell carcinoma of vagina. Here we present a case of forgotten vaginal pessary leading to incarceration.

\section{Case Report}

Mrs Chaitri Devi, 65 yrs, menopausal since 10 yrs resident of small village presented to RIMS outpatient department with complaint of foul smelling discharge per vaginum for 6 months and occasional bleeding PV for last 2 months. She is P6+0, all normal delivery at home with last child birth 20 years back. There was no history of alteration of bladder and bowel habit. She presented as a case of postmenopausal bleeding raising suspicion of genital malignancy.

Her general examination was unremarkable except for mild degree of pallor. On per abdominal examination, no abnormality was detected. Per speculum examination revealed atrophic vaginal wall with evidence of vaginitis. An off white structure of width $1 \mathrm{~cm}$ was visible high up in anterior vaginal wall which raised suspicion of retained vaginal pessary. Our finding got confirmed when a firm ring like structure was felt high up in vagina, lateral and posterior part of which was embedded under fibrosed vaginal epithelium. On further interrogation, she gave history of genital prolapse 10 years back for which she took treatment by local doctor at village and got relieved. She never went there for follow up. Nor the patient or her attendant were able to recall any event when pessary was applied on her.

She was then admitted and preoperative workup was done. All haematological and biochemical parameters were within normal limits. Under iv sedation, anterior part of pessary was held with allis tissue forceps and it was removed after giving incision on thickened vaginal wall over the embedded part of pessary. Fibrosed vaginal wall was then trimmed and opposed by catgut no.0. There was significant oozing from vaginal wall, so tight vaginal packing done by pack soaked with povidone iodine. Pack was then removed after 24 hours and she was discharged with antibiotics prescribed for vaginitis.

Follow-up examination after 1 month showed healthy cervix and vagina but no descend of cervix except for mild cystocoele and enterocoele.

\section{Discussion}

Pelvic organ prolapse is a common cause of morbidity among women of developing countries. Although surgery is the definite treatment, pessaries can give satisfactory result in women who wish or need to avoid surgery. Pessaries are basically of two types- first is support pessaries such as ring pessary which are derived by spring mechanism and thought to be supported by symphysis pubis; another is space filling pessaries which are supported by creation of suction between the pessaries and vaginal walls or by providing the diameter larger than the genital hiatus or by both mechanism. Pessaries are fitted by trial and error mechanism 


\section{International Journal of Science and Research (IJSR) \\ ISSN (Online): 2319-7064 \\ Index Copernicus Value (2013): 6.14 | Impact Factor (2014): 5.611}

but once fitted, proper follow up is must. Ring pessaries which are most commonly used needs 3 monthly checkup.At each visit, patients are asked about any symptoms like vaginal discharge, bleeding, pain and voiding difficulties. Pessaries are then palpated, removed gently and inspection of vaginal wall done for any ulcer or erosions. Any abnormality if found are treated accordingly. Pessaries if uncared may lead to serious complications like erosion, fistula, herniation, uraemia and even carcinoma of vaginal wall.

In the present case, postmenopausal bleeding presented with foul smelling vaginal discharge raising suspicion of genital malignancy but on examination there was a retained foreign body. Forgotten foreign body in vagina is rare, but several cases have been reported. In the present case, the pessary was retained for about 10 years and led to incarceration of pessary within the wall of vagina. Forceful removal of this may lead to torrential bleeding and fistula formation.

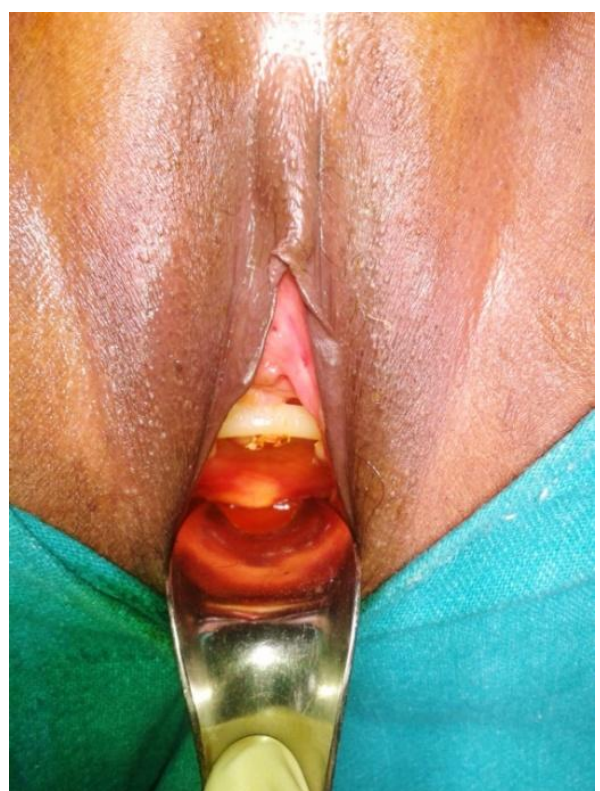

Figure 1: Per speculum examination showing anterior part of pessary.

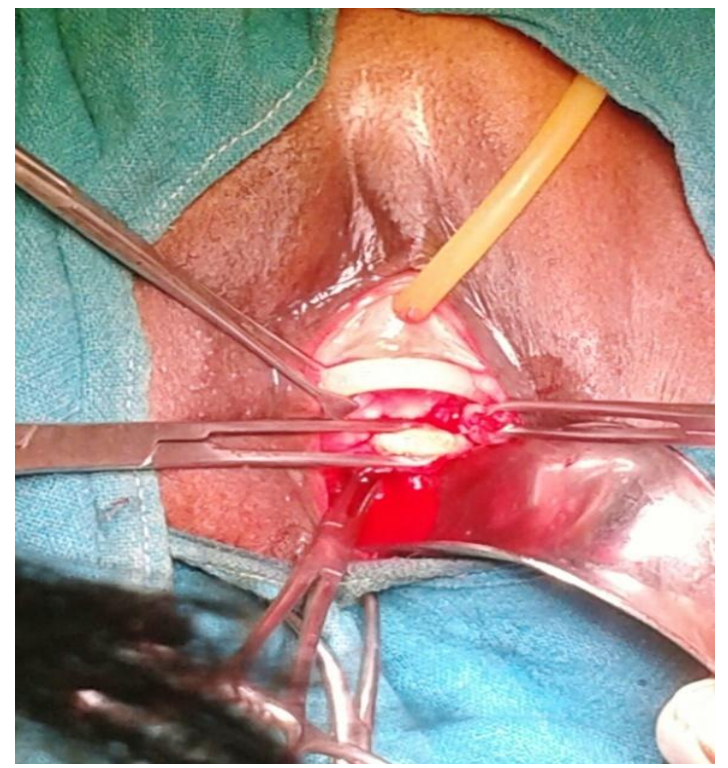

Figure 2: Surgical excision of pessary

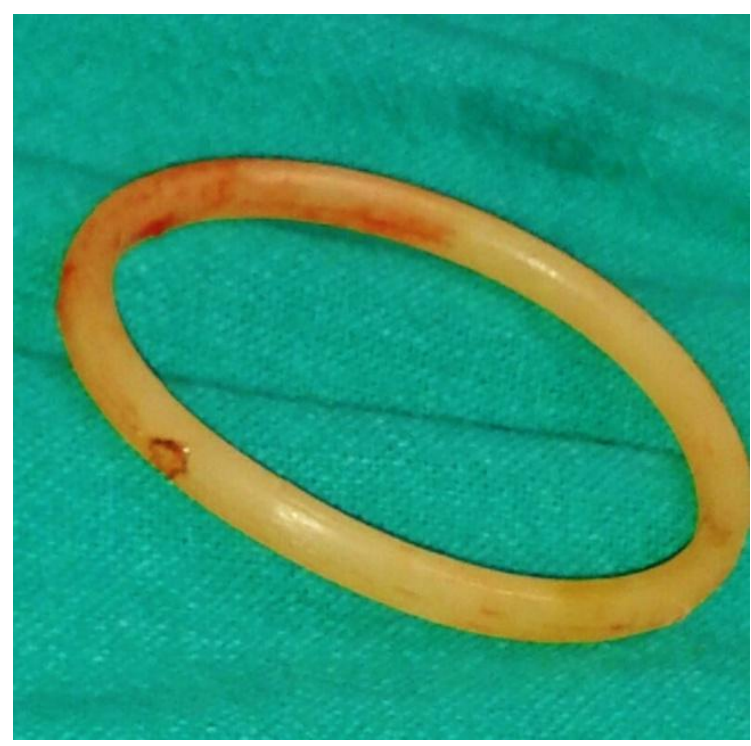

Figure 3: Pessary after removal

\section{References}

[1] Bash KL. Review of vaginal pessaries. Obstet Gynecol Surv 2000;55:455.

[2] Clemons JL, Aguilar VC, Tillinghast TA, et al. Risk factors associated with an unsuccessful pessary fitting trial in women with pelvic organ prolapsed. AM J Obstet Gynecol 2004;190:345.

[3] Emge LA, Durfee RB. Pelvic organ prolapsed: Four thousand years of treatment. Clin Obstet Gynecol 1996;9:997.

[4] Morley GW. Treatment of uterine and vaginal prolapsed. Clin Obstet Gynecol 1996;39:959.

[5] Poma PA. Non surgical management of genital prolapsed: Review and recommendation for clinical practice.J Reprod Med 2000;45:789.

[6] Russel JK. The dangerous vaginal pessary. BMJ 1961;2:1595.

[7] $\mathrm{Wu} \mathrm{V,} \mathrm{Farrell} \mathrm{SA,Baskett} \mathrm{TF,} \mathrm{et} \mathrm{al.} \mathrm{A} \mathrm{simplified}$ protocol for pessary managemaent. Obstet Gynecol 1997;90:990.

[8] Zeitlin MP, Lebhrez TB. Pessaries in geriatric patient. J Am Geriatr Soc 1992;40:635. 